\title{
Poisson-Vlasov Modeling of Parallel Cylinders in Ionospheric Plasmas
}

\author{
Éric Choinière* and Brian E. Gilchrist ${ }^{\dagger}$ \\ University of Michigan, Ann Arbor, Michigan 48109
}

\section{Introduction}

Bare electrodynamic tethers have been proposed for two important space applications: in-orbit propellantless propulsion $[1,2]$ and remediation of Earth-bound radiation belts through the scattering of high-energy electrons leading to their accelerated precipitation [3-5]. The latter application is oftentimes referred to as space remediation.

In both applications, the use of multiple parallel conducting wires is being considered. Such a multistrand structure would provide improved survivability to collisions with micrometeroid. Additionally, since sheath size is roughly a function of the total amount of linear surface charge held on the tether system [6], a multi-wire structure might allow for the formation of the large plasma sheath required for effective scattering in the space remediation application, at a reduced cost in terms of expended power due to the increased capacitance provided by the relative proximity of parallel wires.

In an effort to get a basic understanding of the physics of plasma-immersed multi-wire conductive structures, we consider in this paper a structure consisting of two parallel, identical round cylinders with equal potential bias. Both cylinders have a radius $r_{0}$ and their centers are spaced apart by a distance $\Delta x$.

Section II presents an overview of the theory used in our plasma solvers. Section III presents our simulation results.

\section{Overview of KiPS, a Steady-State Kinetic Plasma Solver}

Self-consistent analyses of the plasma sheath exist [7] which apply to a single round cylinder immersed in a stationary plasma at low bias voltages. Other self-consistent models were developed which accounted for plasma flow $[8,9]$ but applied to a single round cylinder at low bias voltages.

The Kinetic Plasma Solvers (KiPS-1D and KiPS-2D) developed at the University of Michigan are based on a kinetic approach for the self-consistent modeling of a stationary or flowing collisionless unmagnetized plasma in a vast region surrounding any two-dimensional conductive object. Two solvers were developed based on this model:

- KiPS-1D, a 1-D model based on cylindrical symmetry, applicable to round cylinders in stationary plasmas;

- KiPS-2D, a full 2-D model, allowing for arbitrary 2-D geometries in stationary or flowing plasmas.

\footnotetext{
*Graduate Student, Radiation Laboratory, EECS Department, AIAA Student Member, echoinie@umich.edu

†Professor, Electrical Engineering and Space Sciences, AIAA Senior Member, gilchrst@umich.edu
} 
Some of the theory for these models was presented before [10], but will be repeated in part here for completeness. Validation of these models against previously existing models [7-9] has shown excellent agreement for round cylinders over the low voltage regimes covered by those models [6]. In this paper, we use the new simulation capabilities provided by KiPS-2D to simulate the interference effect among parallel wires immersed in a plasma.

\section{A. Poisson-Vlasov Representation of a Collisionless Plasma}

Obtaining a simultaneous solution in two dimensions for the steady-state electric potential and charge density distributions of both plasma species (ions and electrons) requires the ability to solve, self-consistently, Vlasov's equation for each species and Poisson's equation for the electric potential and charge density, while satisfying appropriate boundary conditions.

In charged media such as a plasma, the steady state of the electric potential and net charge distributions obey Poisson's equation [11],

$$
\nabla^{2} V(x, y)=-\frac{\rho(x, y)}{\epsilon_{0}}
$$

at any point within the plasma. In addition, each species comprising the plasma, that is, electrons and ions, is composed of particles with a two-dimensional distribution of velocities at any point in space $f\left(x, y, v_{x}, v_{y}\right)$. The number density of each species is obtained by integrating the velocity distribution function over all velocity space

$$
n_{e, i}=\iint f_{e, i}\left(x, y, v_{x}, v_{y}\right) d v_{x} d v_{y}
$$

where the indices $e$ and $i$ refer to electrons and ions, respectively. The net charge density results from

$$
\rho(x, y)=q_{i} n_{i}(x, y)+q_{e} n_{e}(x, y)
$$

where $q_{e}$ and $q_{i}$ are the electron and ion particle charge.

In a collisionless, unmagnetized plasma at the steady state, the velocity distribution functions $f\left(x, y, v_{x}, v_{y}\right)$ of each species is conserved along particle orbits. This is stated by Vlasov's equation in the steady state $\left(\frac{\partial f}{\partial t}=0\right)[12]:$

$$
\frac{d f_{e, i}}{d t}=v_{x} \frac{\partial f_{e, i}}{\partial x}+v_{y} \frac{\partial f_{e, i}}{\partial y}-\frac{q_{e, i}}{m_{e, i}} \frac{\partial V}{\partial x} \frac{\partial f_{e, i}}{\partial v_{x}}-\frac{q_{e, i}}{m_{e, i}} \frac{\partial V}{\partial y} \frac{\partial f_{e, i}}{\partial v_{y}}=0
$$

where we substituted the electric field components with the gradient of the electric potential, i.e., $\vec{E}=-\nabla V$. Given a known potential distribution and a boundary condition for the incoming particles at the outer boundary of the computational domain, the method of characteristics can be used to solve (4) for the velocity distributions $f_{e, i}\left(x, y, v_{x}, v_{y}\right)$ of both the electrons and ions, at all positions and for all velocities.

The use of the method of characteristics for the resolution of Vlasov's equation in plasma problems was reported early on [13], and was referred to as the inside-out procedure. It consists of tracking a particle's trajectory back in time until it intersects the "source boundary" of computational space, where the velocity distribution function is sampled. Any trajectory not originating from the outer shell is deemed unpopulated [7]. Such is the case for trajectories originating from the tether itself, which we assume does not emit charged particles, as well as trapped trajectories, which have no sources in the collisionless case.

The steady-state of the plasma must simultaneously satisfy both Poisson's equation (1) and Vlasov's equation (4), subject to appropriate boundary conditions on the potential and the incoming particles.

\section{B. Regularized Newton-Iterative Poisson-Vlasov Solver}

To find the steady-state solution, both a Poisson solver and a Vlasov solver are implemented: 
- The Poisson solver computes a numerical approximation for the potential distribution $V(x, y)$ based on the finite element method $[6,14]$, given a net charge distribution $\rho(x, y)$ together with appropriate floating boundary conditions on the potential distribution, consistent with Poisson's equation (1). The finite element implementation is based on a piecewise-bilinear representation of potential and charge density distributions over an unstructured set of triangles covering the computational domain. Details of the 1-D and 2-D implementations of the Poisson solver are given in ref. 6 .

- the Vlasov solver computes a numerical approximation for the charge distribution $\rho(x, y)$, given a potential distribution $V(x, y)$, consistent with Vlasov's equation (4) as well as with (2) and (3). The density calculations are computed by integrating samples of the velocity distribution function over velocity space. Using reverse-time trajectory tracking, these samples can be inferred from the known velocity distribution functions of the background plasma (the source) by virtue of the fact that the velocity distribution function $f\left(x, y, v_{x}, v_{y}\right)$ is conserved along trajectory orbits in collisionless plasmas. This technique is known in mathematics as the method of characteristics [15], or, alternatively, as the inside-out procedure as introduced by Parker [13] for plasma simulations.

The steady-state solution lies at the fixed point of the combined PoissonVlasov operator enclosed within the dashed box on Figure 1. Now, there are known difficulties arising in solving such a problem [7]. Simple iteration of the fixed point operator does not in general yield convergence, since it is a non-contractive mapping [16]. To address these difficulties, a procedure was implemented based on Newton's method for nonlinear systems of equations [17]. This technique, which we designate as the Progressive Tikhonov-regularized Newton iterative procedure, is depicted in Figure 1. It consists of progressively improving a solution vector $\vec{\rho}_{\text {in }}$, i.e., reducing the residual norm $\left\|\vec{\rho}_{\text {in }}-\vec{\rho}_{\text {out }}\right\|$, by successively linearizing the Poisson-Vlasov operator and finding a regularized solution for the resulting linearized system of equations. This process relaxes the solution vector $\vec{\rho}_{\text {in }}$ and has the global effect of reducing the Euclidean distance between $\vec{\rho}_{\text {in }}$ and $\vec{\rho}_{\text {out }}$. The iterative process continues until the solution can no longer be

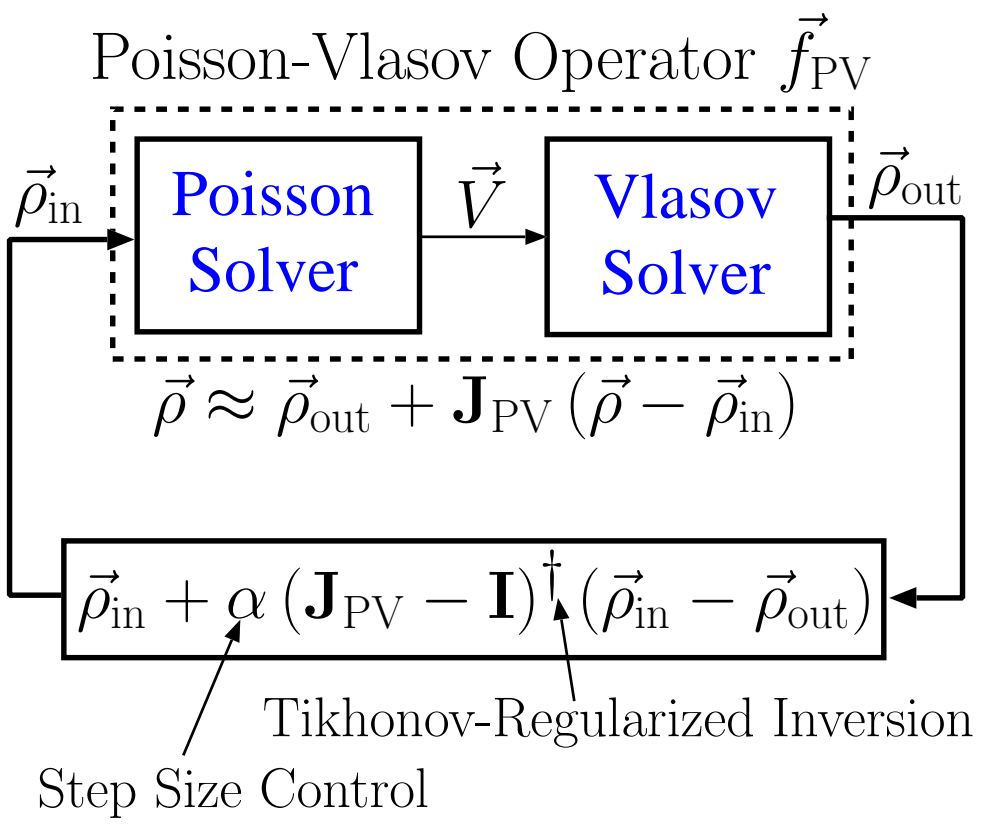

Figure 1. Progressive Tikhonov-regularized Newton iterative Poisson-Vlasov procedure. $\mathbf{J}_{P V}$ is a Jacobian matrix containing the linearized behavior of the Poisson-Vlasov operator near an "operating point" $\vec{\rho}_{\text {in }}$.

improved with the specified quadrature accuracy used in the Vlasov solver. In practice, a suitable quadrature accuracy is chosen that allows the iterates to reach a reasonably low residual norm $\left\|\vec{\rho}_{\text {in }}-\vec{\rho}_{\text {out }}\right\|_{\infty}$, on the order of $1 \%$ of the background plasma density.

The necessity of the Tikhonov regularization stems from the ill-conditioning of the linearized system, which tends to allow quadrature noise into the solution, causing rapid divergence of the iterates. The regularization process prevents "oversolving" the linearized system at any given iteration, making the procedure tolerant to ill-conditioning and quadrature noise [6].

Figure 2 depicts the flow of computed quantities within a typical KiPS-2D run, at the converged state. The best estimate for the input net charge density profile $\rho_{\text {in }}(x, y)$ is used to compute the electric potential 


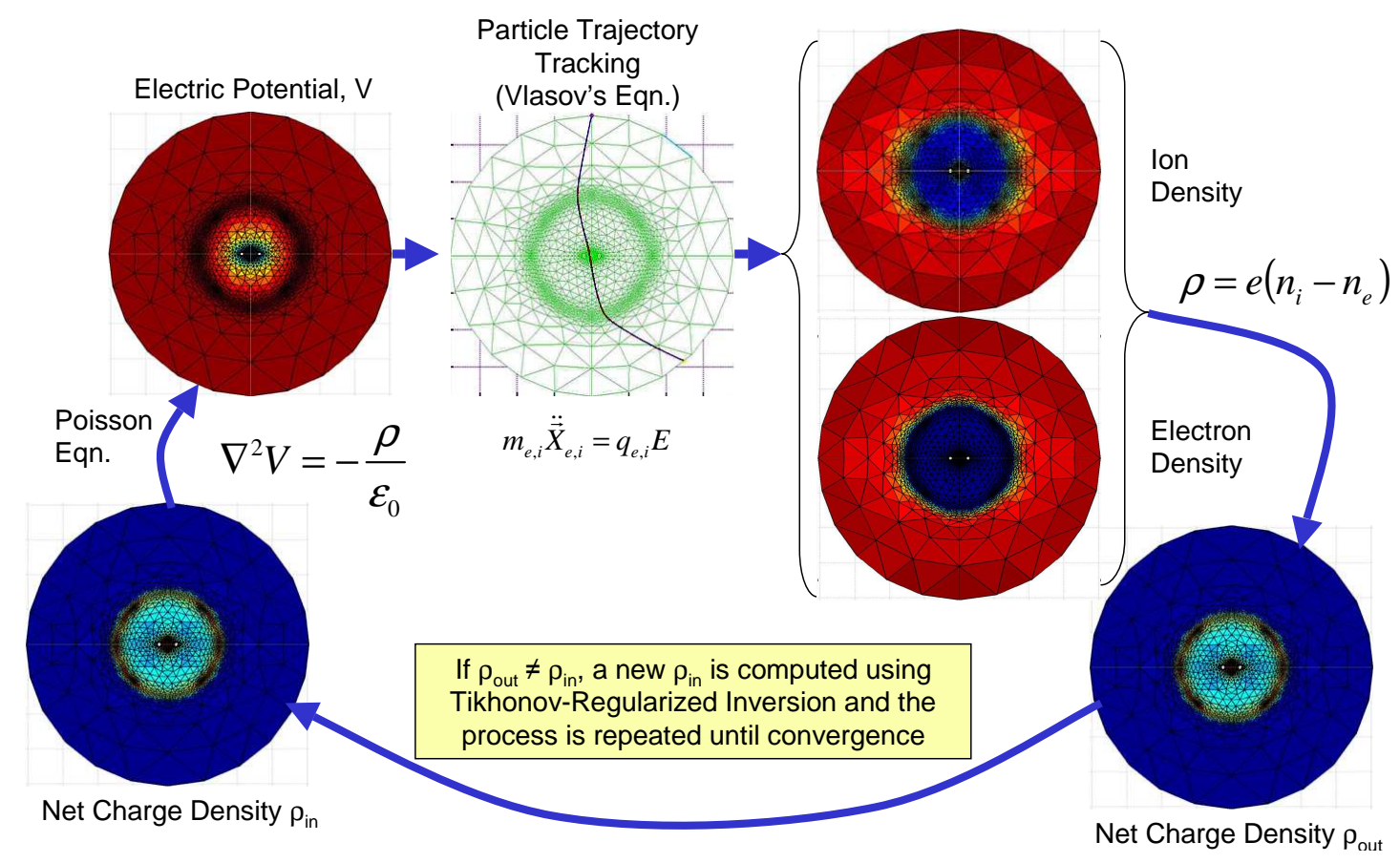

Figure 2. Illustration of the Converged State of the Iterative Resolution Process Used to Find the SelfConsistent Steady-State Solution. This specific solution corresponds to a simulation of two parallel wires.

profile $V(x, y)$ from Poisson's equation (1). Vlasov's equation is then solved using particle trajectory tracking (i.e., the Vlasov solver) to infer the ion and electron density profiles $n_{i}(x, y)$ and $n_{e}(x, y)$ that are consistent with the obtained potential profile. Finally, the net charge density profile $\rho_{\text {out }}(x, y)$ is inferred from the ion and electron density profiles using (3). On Figure 2, the very low discrepancy between $\rho_{\text {in }}(x, y)$ and $\rho_{\text {out }}(x, y)$ indicates that we have reached a solution corresponding to the steady-state of the plasma profile.

\section{KiPS-1D and KiPS-2D: Implementations Compared}

Table 1 compares various aspects of the computer implementation of the 1-D and 2-D solvers. The primary difference between both implementations is that KiPS-1D runs on a single CPU, whereas KiPS-2D has a parallel implementation. While the 1-D implementation of the Vlasov solver does not require trajectory tracking, KiPS-2D's Vlasov solver requires tracking about 10,000 particle trajectories per density sample at any given iteration, which totals to a few tens of millions of trajectories per iteration. This can only be accomplished in a reasonable time using some form of parallelism. The 2-D version of the Vlasov solver was thus implemented in Fortran 90 using an MPMD ${ }^{a}$ parallel processing scheme based on the Parallel Virtual Machine library [18]. Since the Vlasov solver consists of computing a fixed number (say $M$ ) of plasma density samples throughout the computational domain based on a given potential profile, it can easily be divided into $M$ independent sub-tasks. These sub-tasks are distributed among the slave nodes on a parallel platform in what constitutes a coarse-grained parallel algorithm.

A dynamically-configured pool of about 150 Sun Blade 1000/1500 workstations, each acting as a slave node as part of the Vlasov solver, is used as the parallel platform. A total of 250 workstations are being used on an opportunistic basis, being swapped in and out of the 150-workstation slave node pool according to their availability. ${ }^{\mathrm{b}}$

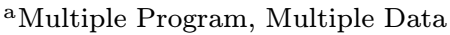

${ }^{\mathrm{b}} \mathrm{A}$ workstation is removed from the slave node pool when a console user logs in, and only moved back in when no console
} 


\begin{tabular}{|l|l|}
\hline \multicolumn{2}{|c|}{ KMPLEMENTATIONS COMPARED } \\
\hline \hline Kerial Poisson and Vlasov solvers & \multicolumn{1}{c|}{ KiPS-2D } \\
\hline $\begin{array}{l}\text { Management of top-level iteration routine and } \\
\text { Poisson Solver under Matlab }{ }_{\mathrm{TM}} ;\end{array}$ & $\begin{array}{l}\text { Serial Poisson solver, Parallel Vlasov solver; } \\
\text { Poisson Solver under Matlab }\end{array}$ \\
\hline $\begin{array}{l}\text { Vlasov Solver implemented in Fortran 90 for } \\
\text { computational efficiency; }\end{array}$ & $\begin{array}{l}\text { Vlasov Solver implemented in Fortran 90, runs } \\
\text { on multiple slave nodes - density samples are } \\
\text { computed by individual nodes; }\end{array}$ \\
\hline Runs on a single PC; & $\begin{array}{l}\text { Parallel Virtual Machine library used to harness } \\
\text { a dynamic cluster of 150 Sun Blade Workstations }\end{array}$ \\
\hline Run times from a few seconds to a few minutes & Run times from a few minutes to a few hours \\
\hline
\end{tabular}

Table 1. Comparison of the KiPS-1D and KiPS-2D implementations of the self-consistent steady-state kinetic model

\section{Duality of ion- and electron-attracting configurations}

In the negative-bias situation under consideration, the ions are the attracted species. Since the plasma is not moving (no flow), the results we obtain are also directly applicable to the electron-attracting situation with equal bias magnitude, provided that we swap the ion and electron density profiles.

\section{E. Treatment of Repelled Electrons}

Our model allows for the full kinetic representation of both species. However, in a stationary situation we know that the electric potential will have the same sign as the potential bias, everywhere in space around the perturbation, and certainly everywhere within the computational zone. For a repelling bias of such a large magnitude as that considered here $\left(\phi_{0}=-320\right)$, we can affirm that, for all practical purposes, none of the electron trajectories which contribute to the electron density intersect the conductive cylinders. This means that all electron trajectories are connected to the background plasma, and allows us to use with excellent accuracy the Boltzmann equation for the electron density:

$$
n_{e}=n_{0} \exp \frac{V}{T_{e}}
$$

where we assume that $V<0$ everywhere in space. This approach results in significant computational savings, due to the fact that only the ions need to be treated kinetically.

\section{Simulation Results for Two Parallel Cylinders}

The geometrical parameters are the cylinder radius $r_{0}$ and the center-to-center spacing $\Delta x$. All of the simulation results presented in this section were performed using a cylinder radius $r_{0}=\lambda_{\text {De }}$ and a normalized potential bias $\phi_{0}=\frac{V_{0}-V_{p}}{T_{e}}=-320$.

We consider the effect of the center-to-center spacing on sheath structure and current collection, always comparing performances to those of a single round cylinder with radius $r_{0}=\lambda_{\text {De }}$ and bias $\phi_{0}=-320$. The value of the center-to-center spacing $\Delta x$ varies from $\Delta x=\lambda_{\text {De }}$ (cylinders touching) to $\Delta x=300 \lambda_{\text {De. This }}$ maximum spacing corresponds to about 16 times the single-wire effective sheath radius of $19 \lambda_{\text {De }}[6]$.

users are logged in. 


\section{A. Orbits of the Attracted Ions}

We now turn our attention to the orbits of the attracted ions. In the following we discuss the existence of complex ion orbits which do not exist in the single-cylinder case, and explain why the criteria used to determine whether an orbit is trapped (and unpopulated) must be less restrictive than in the case of the single cylinder. We then show some examples of these complex orbits.

\section{Criteria for Trapped Orbits}

The symmetric potential structure which exists in the simple single-cylinder case does not allow any of the non-trapped trajectories to feature more than one change of "radial direction", that is, one change of sign of the radial component of velocity, $v_{r}$. In other words, any orbit originating from the background plasma will either:

- approach the conductive cylinder $\left(v_{r}<0\right)$, miss it due to excessive angular momentum and return to the background plasma $\left(v_{r}>0\right)$;

- approach the conductive cylinder and get collected onto it ( $v_{r}<0$ all along).

When two parallel cylinders immersed in a plasma are placed sufficiently close to one another (i.e., when the individual sheath radius is a non-negligible fraction of the center-to-center spacing $\Delta x$ ), the shadow effect created by one cylinder can affect the space charge surrounding the other sufficiently to create asymmetries in the potential structure. Those asymmetries, in turn, could allow for the existence of collisionless trajectories of an increased complexity, featuring for example several "radial oscillations" about a given cylinder, or even "figure eight" trajectories orbiting about both cylinders. At any given location, some of the directions in velocity space that were unpopulated when there was no neighboring cylinder may now be populated through relatively complex paths originating from the background plasma, while other directions that were previously populated may now be unpopulated due to the existence of new types of trapped orbits, such as the "figure eight".

As a consequence, we need to allow for a sufficiently large number of radial oscillations to accommodate for the existence of these more complex orbits, while at the same time keeping it down to a reasonable value to obtain reasonable simulation times. We have found that allowing for up to 20 radial oscillations provided such a compromise.

\section{Examples of Ion Orbits}

In Figure 3 we show a few typical examples of ion orbits through the self-consistent potential structure of the two-cylinder configuration with a center-to-center spacing $\Delta x=20 \lambda_{\mathrm{De}}$, a potential bias $\phi_{0}=-320 T_{e}$ and a cylinder radius $r_{0}=\lambda_{\mathrm{De}}$. The circle shown at one end of each trajectory indicates the location of the interrogation point where the velocity distribution function is being sampled. Following the trajectory from this point backward in time leads us to the "source" point of the trajectory, indicated by a square.

The source point can either be the background plasma, a cylinder's surface (in which case the orbit is unpopulated since the cylinder is not a source), or it may be undetermined in the case of a trapped (unpopulated) orbit.

Figures 3(a)-3(c) have a common interrogation point, located on a node on the right side of the mesh. Figure 3(a) shows an example of an unpopulated orbit originating from the surface of the rightmost cylinder; this orbit is not populated because the cylinder is not a plasma source. Figure 3(b) depicts a populated orbit that undergoes two loops around the rightmost cylinder before reaching the interrogation point. Figure 3(c) depicts a very complex but populated ion orbit that originates from the background plasma, undergoes several loops around both cylinders, and finally reaches the interrogation point.

Figure 3(d) corresponds to a different interrogation point. It is shown here to illustrate one example of an unpopulated orbit that was deemed "trapped", having exceeded the maximum number of radial oscillations set for this simulation, which was set to 20 . 


\section{B. Inspection of the 2-D Sheath Structure}

We now consider the general aspect of the sheath structure surrounding both cylinders for a few values of the center-to-center spacing: $\Delta x=2 \lambda_{\mathrm{De}}, 10 \lambda_{\mathrm{De}}, 20 \lambda_{\mathrm{De}}$, and $80 \lambda_{\mathrm{De}}$. First, we note that all of the twocylinder simulations presented here were performed using two axes of symmetry. In other words, only a single quadrant had to be simulated, thanks to the symmetry of the two-cylinder geometry and the fact that the plasma is not flowing. The density distributions shown here, however, are shown over two quadrants, in order to clearly illustrate the two-cylinder geometry under consideration. The simulation results, spanning the quadrant $\theta \in[0, \pi / 2]$, were simply "mirrored" to the second quadrant in $\theta \in[\pi / 2, \pi]$. We have opted to only show the results in the half-space $\theta \in[0, \pi]$, so that the features of interest near the cylinders can be clearly seen. All of the distributions shown in Figures 4 through 7 are given in the following two formats:

- 2-D distributions are shown on the left, over the half-space $\theta \in[0, \pi]$;

- one-dimensional profiles of cross-sections performed along the $x$ axis are shown on the right.

Figure 4 shows the electron and ion density distributions pertaining to the smallest center-to-center spacing, $\Delta x=2 \lambda_{\mathrm{De}}$ (cylinders touching). Due to the proximity of the two cylinders, the profiles obtained are very close to what would be obtained with a single cylinder. The ion density, shown on Figure 4(b), is seen to drop to just under half the ambient density on the external surfaces of both cylinders, an indication that the total extent of the two touching cylinders is barely large enough to create a departure from the orbital motion limit of half the ambient density. Radial acceleration of ions is responsible for the significant drop in ion density as the ions enter the sheath, a phenomenon that is also observed in the single-cylinder case [6].

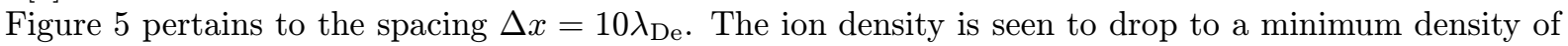
about $0.2 n_{0}$ as the ions enter the sheath. A relatively large ion density is seen to exist in between the two cylinders, where it reaches a maximum of about $0.7 n_{0}$. The ion density at the surfaces of the cylinders is seen to be much lower here than what was seen for the case where the cylinders are touching. The ion densities on the external and internal surfaces of the cylinders are about $0.27 n_{0}$ and $0.19 n_{0}$, respectively. This is an indication that the orbital motion limit was not achieved for current collection, due to the overall extent of this "two-cylinder" probe. The center-to-center spacing is not quite large enough for the two sheaths to have separated, so that the two cylinders are in effect emulating a larger structure, leading to reduced current collection with respect to OML, similar to what is seen as the radius of a single cylinder goes from a fraction of a Debye length to several Debye lengths [19].

Figure 6 pertains to the spacing $\Delta x=20 \lambda_{\text {De. }}$. Here the ion density profile shows various "bumps" as we enter the sheath, which may be due to the partial filling of some of the velocity space's directions that were left empty in the single-cylinder case due to angular momentum restrictions. Some of these directions are being populated by the complex trajectories described earlier. The ion acceleration through the sheath still results in a significant drop of the ion density, albeit for a somewhat smaller range of distances from the cylinder's surface. The ion density at the surface is still well below the orbital motion limit: we have $n_{i} \approx 0.2 n_{0}$ on the inside surface and $n_{i} \approx 0.3 n_{0}$ on the outside surface. We can therefore still expect a significant current reduction as compared to the OML limit. The peak in ion density previously seen for $\Delta x=10 \lambda_{\text {De }}$ has dropped to about $n_{i} \approx 0.58 n_{0}$, as the sheath has begun to separate into two separate sheaths, which causes some restrictions of the angular momentum with respect to each cylinder's axis.

Figure 7 pertains to the spacing $\Delta=80 \lambda_{\text {De. }}$ At this large spacing, the two cylindrical sheaths have now separated, and the electron density now peaks at above half of the ambient density in between the two cylinders. We now distinguish a net charge distribution which features two structures resembling the net charge distribution around a single independent cylinder, except for some remaining strong asymmetry. This asymmetry remains in spite of the fact that the surface charges located on one cylinder practically no longer contribute to the electric fields within the sheath around the other cylinder. Rather than being due to a direct field coupling, the remaining asymmetry is due to the fact that some velocity space directions in the sheath around one cylinder are left unpopulated because their corresponding trajectories originate from

7 of 20

American Institute of Aeronautics and Astronautics 
the surface of the other cylinder. This effect can be significant because of the focusing effect between the two sheaths which concentrates trajectories toward the inside of the sheath even as they are moved apart significantly. The asymmetries are also amplified by the changes that they induce in the population of the orbits that are normally "trapped and unpopulated" in the case of an independent cylinder. The ion density profile seen on Figure 7 shows two familiar patterns resembling the ion density profile typical of a single independent cylinder [6], albeit with some asymmetry. The ion density reaches a value of about $0.54 n_{0}$ on the $x$ axis in between the two cylinders. We observe that this is a significantly lower density than would be observed at such a large distance from an independent cylinder (about $0.75 n_{0}$ ). The asymmetry however causes some increase of the "outside" density profile, where we observe a "bump".

The observations that we have made concerning the widest spacing can be summarized as follows:

1. the ion densities on the outermost side of a given cylinder are somewhat increased by the potential asymmetries caused by the trajectory connections among both sheaths;

2. the ion densities on the innermost side of a given cylinder are somewhat decreased due to the unpopulated trajectories originating from the surface of the other cylinder.

While the first observation may lead to a decrease of the effective sheath radius as measured on the outermost side as compared to that of an independent cylinder, the second observation may cause an increase of the "inner" effective sheath radius. The overall outcome of these two competing effects is difficult to predict. We will assess it in the following two sections based on a more general measure of the sheath dimensions, the effective sheath area.

\section{Definition of an Effective Sheath Area Concept}

A useful first-order metric for the scattering efficiency of the sheath structure is that of the effective sheath area $A_{s}$, which in the case of a single independent cylinder relates to an effective sheath radius $r_{s}$ through $A_{s}=\pi r_{s}^{2}$. For a single independent cylinder, the effective sheath radius $r_{s}$ is defined based on the asymptotic form of the potential profile,

$$
\phi(r)=\phi_{0}\left(1-\frac{\ln \left(r / r_{0}\right)}{\ln \left(r_{s} / r_{0}\right)}\right) .
$$

This asymptotic potential profile most accurately describes the potential drop near the cylinder's surface, and drops to zero faster than the fully self-consistent solution of the potential, which comprises a tail that extends the potential structure further out. This asymptotic description represents the equivalent problem of a coaxial capacitor, with all of the space charge concentrated on a single radius $r=r_{s}$.

We now seek to generalize the concept of effective sheath radius for application to non-symmetric sheaths, while providing a metric consistent with that used for round cylinders.

Let us first consider a sheath with circular symmetry, for which (6) applies. We define an effective sheath area $A_{s}$ consistent with our previous definition of the effective sheath radius $r_{s}$ based on the relationship

$$
A_{s}=\pi r_{s}^{2}
$$

where we include the area of the cylinder itself as part of the effective sheath area. Similarly, we define

$$
A=\pi r^{2} \quad \text { and } \quad A_{0}=\pi r_{0}^{2},
$$

where $A$ is the area enclosed by any equipotential circle of radius $r$, and $A_{0}$ is the cylinder's cross sectional area. Using (7) and (8), we re-write the potential profile given by (6) in terms of $A_{s}, A$ and $A_{0}$ :

$$
\phi=\phi_{0}\left(1-\frac{\ln \left(A / A_{0}\right)}{\ln \left(A_{s} / A_{0}\right)}\right) .
$$

$$
8 \text { of } 20
$$


This asymptotic relationship relating the potential on any contour level to the area it encloses, holds from the surface of the cylinder out to a fairly large radius $r$ (or area $A$ ).

We may now generalize (9) to any type of sheath structure, by considering the variation of the potential $\phi$ as a function of the area $A$ enclosed within the corresponding contour level, and finding an appropriate measure of the sheath area $A_{s}$ based on some asymptotic behavior of the $\phi$-vs- $A$ plot. As was just demonstrated, in the case of the single round cylinder, this definition will provide us with a measure for the effective sheath area $A_{s}$ consistent with the effective sheath radius $r_{s}$ defined earlier for a single independent cylinder.

\section{Determination of the Effective Sheath Area of the Two-Cylinder Configuration}

Figure 8 graphically illustrates, for a spacing $\Delta x=10 \lambda_{\text {De }}$, the process that was used to determine an effective sheath area $A_{s}$, consistent with the definition given in Section C. Part (a) shows a set of equipotential contour levels and part (b) shows a plot of the potential $\phi$ as a function of the surface area $A$ enclosed by the corresponding contour level. The effective sheath area $A_{s}$ is determined by the $A$-intercept ${ }^{\mathrm{c}}$ of the extrapolated asymptotic behavior of the $\phi$-vs- $A$ data. In the particular case shown in Figure 8 , the twocylinder effective sheath area is $A_{s}=2244.6 \lambda_{\mathrm{De}}^{2}$, which compares to $A_{s, 1}=\pi r_{s}^{2}=1128.6 \lambda_{\mathrm{De}}^{2}$ for a single independent cylinder.

The semilog graph of the $\phi$-vs- $A$ data shows two distinct linear regions, indicated by separate linear "best fits". The first linear region (on the left) corresponds to the set of circular contour levels enclosing each cylinder separately. The slope associated with this first region is not as steep as that which would be expected of an independent cylinder, and is consistent with the observed lower amount of normalized surface charge $Q_{n}=Q /\left(\epsilon_{0} T_{e}\right)$, where $Q$ is the surface charge in Coulombs, held separately by each of the cylinders as compared with the surface charge held by an independent cylinder. ${ }^{\mathrm{d}}$ This lower charge can be attributed to a "virtual" effective sheath edge enclosing an area $A_{s \text {,virtual }}$ (shown on the figure to be further out to the right of the graph) that is much larger than the effective sheath area of an independent cylinder. The fact that each cylinder holds a lower amount of surface charge than an independent cylinder is consistent with the large area $A_{s, \text { virtual, }}$, as is evidenced by the following equation applicable to coaxial capacitors [11]:

$$
Q_{n, 1}=4 \pi \frac{\phi_{0}}{\ln \left(A_{s} / A_{0}\right)}
$$

where $Q_{n, 1}$ is the normalized surface charge held by each cylinder and $A_{0}=\pi r_{0}^{2}$ is the single-cylinder area. The expression given by (10) clearly shows that the surface charge $Q_{n, 1}$ goes down with increasing sheath area $A_{s}$.

The leftmost linear region extends until we approach a critical point where the equipotential contours of both cylinders connect together to form a single contiguous contour. The $\phi$-vs- $A$ data then rapidly switches to the rightmost linear region corresponding to the set of circular contour levels enclosing both cylinders. The effective sheath area of the system is defined as the $A$-intercept of the rightmost linear fit. In this particular case, it turns out that the effective sheath area $A_{s}$ is almost exactly equal to the combined sheath areas of two independent cylinders, i.e., $A_{s} \approx 2 A_{s, 1}$.

Space charge effects can be seen at two different locations on Figure 8. The first one occurs near the inflection point between both linear regimes, where the $\phi$-vs- $A$ data is seen to momentarily drop below the leftmost linear best-fit before it starts growing faster toward the rightmost linear best-fit. This momentary slope reduction is attributed to the shielding effect of space charge, which is having a detectable effect because of the sufficient spacing between the cylinders, where some space charge exists. Beyond the turning point of this graph, the effect of space charge becomes negligible again for a large portion of this outer sheath, until we reach beyond an area $A \approx 1000 \lambda_{\mathrm{De}}^{2}$, where we notice the typical tail-like behavior of the potential associated with space charge shielding.

\footnotetext{
cThe $A$-intercept is defined as the value of $A$ at which the linear graph intersects the $A$ axis.

d Note that even though the surface charge $Q_{n}=1052.5$ shown on Figure 8 is larger than the surface charge held by an independent cylinder $Q_{\mathrm{n}, 1}=683.4$, the amount of charge held individually by each cylinder, $\frac{1}{2} Q_{n}=526.25$, is lower.
} 


\section{E. Parametric Analysis of the Sheath Structure}

Using the definitions given in the previous section, we can now consider an analysis of the effects of the center-to-center spacing on the effective sheath area and total surface charge held by the cylinders.

First we define the effective sheath area ratio as the ratio of the total effective sheath area $A_{s}$ of a two-cylinder system to the effective sheath area of a single independent cylinder, $A_{s, 1}$, i.e.,

$$
R_{A_{s}} \equiv \frac{A_{s}}{A_{s, 1}}
$$

Figure 9 depicts the variation of the effective sheath area ratio $R_{A_{s}}$ as a function of the center-to-center spacing $\Delta x$ of the two parallel cylinders.

The leftmost data point in Figure 9 corresponds to a spacing of $\Delta x=2 \lambda_{\text {De }}$ and applies to "touching" cylinders since they both have a radius $r_{0}=\lambda_{\text {De }} . R_{A_{s}}$ is seen to start around 1.55 when the cylinders are touching, indicating a total sheath area $55 \%$ larger than that of a single cylinder. For a smaller cylinder radius, we would expect to obtain $R_{A_{s}}=1$ when the cylinders are touching because the full extent of the two touching cylinders would be small enough for the shadowing effect to be negligible on the density of outward-moving ions throughout the sheath [6].

The ratio $R_{A_{s}}$ then rises to a maximum very close to 2.0 for a spacing $\Delta x=20 \lambda_{\text {De }}$, indicating that the effective sheath area is now on par with the sheath area expected of two independent cylinders. This represents an optimal spacing for purposes of maximizing the total sheath area, as is desired for the highenergy particle precipitation application. This optimal spacing is followed by a sharp drop of the ratio $R_{A_{s}}$ to a minimum value at a spacing $\Delta x=40 \lambda_{\mathrm{De}}$. The sheath area then goes back up relatively quickly until the spacing reaches $\Delta x=60 \lambda_{\mathrm{De}}$, and then follows a slow upward slope for the remainder of the graph. It is seen that the total effective sheath area has only reached about 1.5 times the sheath area of a single independent cylinder at a spacing of $\Delta x=200 \lambda_{\mathrm{De}}$, whereas we expect it to reach a ratio $R_{A_{s}}=2.0$ when the spacing is sufficient for the two sheaths to be fully independent of each other. An extrapolation of a linear fit performed on this slow increase indicates that "full independence" would be reached at an approximate spacing $\Delta x \approx 660 \lambda_{\text {De }}$, which corresponds to about 35 times the independent cylinder sheath radius of $R_{s} \approx 19 \lambda_{\mathrm{De}}$.

The surface charge ratio is shown in Figure 10. This ratio is defined as the ratio of the total surface charge held by both cylinders to the surface charge held by a single independent cylinder. As discussed before, thinner cylinders allowing reduced spacings would carry the same amount of charge as a single thin cylinder when approached sufficiently close to one another. Since here we are considering relatively large cylinders with a radius of one Debye length $\left(r_{0}=\lambda_{\text {De }}\right)$, they cannot be brought sufficiently close together to achieve as low a capacitance as a single wire. This is why the graph shown on Figure 10 starts above 1.0, at a ratio of about 1.1 for a spacing $\Delta x=2 \lambda_{\mathrm{De}}$. As the cylinders are spaced apart, their combined capacitance increases so that the amount of charge they hold grows to a value of about 2.25 times the single-

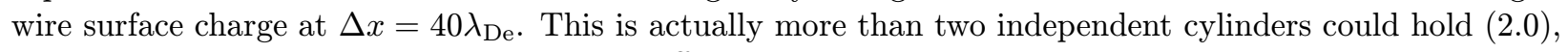
and is consistent with the observed reduced effective sheath area seen in Figure 9 as compared to the total sheath area of two independent cylinders. The total charge then slowly ramps down as the spacing increases beyond $\Delta x=40 \lambda_{\mathrm{De}}$, and the graphed ratio should eventually reach 2.0 .

Figure 11 is an alternative representation of the information shown in Figure 9. It shows the "equivalent potential bias" for a single cylinder. This is the potential bias required on a single cylinder to generate an effective sheath area equal to that generated by our two-cylinder system biased at $\phi_{0}=-320 T_{e}$. It is seen that one could create a sheath as large as that which is created by a single cylinder of radius $r_{0}=\lambda_{\mathrm{De}}$ biased at $\phi_{0}=-570 T_{e}$ by using two cylinders of equal radius $r_{0}=\lambda_{\text {De }}$ biased at $\phi_{0}=-320 T_{e}$ and spaced $20 \lambda_{\mathrm{De}}$ apart. 


\section{F. Interference Effect on Collected Current}

Figure 12 depicts the variation of collected ion current as a function of center-to-center spacing. The graph is normalized to the current that would be collected by two independent cylinders, $I_{\text {indep }}$. In the present case, which involves cylinders with a relatively small radius $r_{0}=\lambda_{\text {De }}, I_{\text {indep }}$ is very close to the orbital motion limit $[6]$.

When the cylinders are touching $\left(\Delta x=2 \lambda_{\text {De }}\right)$, the current ratio $I / I_{\text {indep }}$ is about 0.79 . This relatively low ratio is attributable primarily to the concave structure formed by the two cylinders, which prevents many incoming directions from reaching the facing internal surfaces of the two cylinders. In fact, one can approximate the current that should be collected based on the current entering a convex envelope enclosing both cylinders, as is shown on Figure 13. If we assume that the overall two-cylinder structure is still sufficiently small for orbitalmotion-limited current collection to apply, we can compute the expected collected current based on the ratio of the convex envelope's perimeter to the concave perimeter [20] as follows:

$$
I_{\text {expected }}=I_{\mathrm{oml}} \times \frac{\text { convex perimeter }}{\text { concave perimeter }}=\frac{4 r_{0}+2 \pi r_{0}}{4 \pi r_{0}} \approx 0.82 .
$$

This value (0.82) is just above our result of 0.79 . The remaining discrepancy can be attributed to a mild departure from the OML limit due to the overall size of the collecting structure. This is supported by the fact that the ion density at the external surface of the cylinders is slightly lower than half the

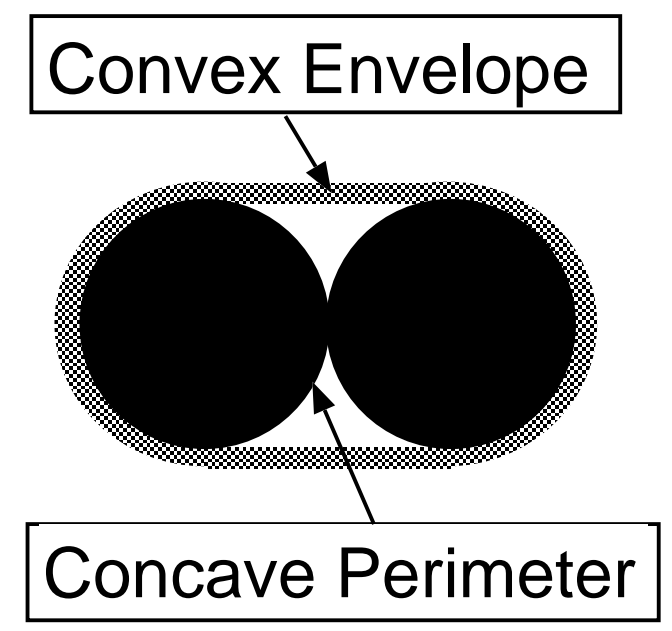
ambient density, as seen on the $x$ axis cross-section profile of Figure 4(b).

As the cylinder spacing is increased, the collected ion current is seen to drop to a minimum just below half of the independent cylinder current near an optimal spacing of $\Delta x=10 \lambda_{\text {De }}$. The current then rises sharply at a spacing $\Delta x=30 \lambda_{\text {De }}$, when two separate sheaths have begun to form. The remainder of the graph shows a rather steady increase of collected current with increasing spacing. This steady increase occurs once the sheaths have completely separated and are primarily coupled through the empty ion orbits connecting both cylinders' surfaces. The observed current increase is attributed to the gradual reduction of the number of connected ion orbits, which are not populated and therefore do not contribute current at the cylinders' surfaces. It is interesting to note that a similar observation was made as part of a separate experimental effort [21] concerning the experimentally measured current collected by slotted tape samples. The correlation between the experimentally observed decrease in the collection efficiency of the perpendicular slotted tapes with increasing gap widths and our simulation results indicate that the gap spacings sampled during our experiments were all located on the left of the expected minimum point on the current-vs-spacing graph.

\section{Conclusion}

KiPS-2D was used to model the interaction of two parallel conductive cylinders (tethers) in a plasma. Interference effects between two parallel cylinders were shown to exist for spacings upward of 20 times the single-cylinder sheath radius, and an optimal spacing of about half the single-cylinder sheath radius was seen to maximize the overall sheath area to a value equal to that of two independent single-cylinder sheaths. Near the same optimal spacing, current collection is reduced to a minimum of half of the current collected by two independent cylinders. This is attributed to the existing set of unpopulated collisionless paths connecting both cylinders. 


\section{Acknowledgments}

The authors would like to thank Tethers Unlimited, Inc. for support of this work, as well as the University of Michigan's Computer Aided Engineering Network for providing the computational resources necessary for this modeling effort.

\section{References}

\footnotetext{
${ }^{1}$ Sanmartín, J., Martínez-Sánchez, M., and Ahedo, E., "Bare Wire Anodes for Electrodynamic Tethers," Journal of Propulsion and Power, Vol. 9, No. 3, 1993, pp. 353-360.

${ }^{2}$ Estes, R., Lorenzini, E., Sanmartín, J., Peláez, J., Martínez-Sánchez, M., Johnson, C., and Vas, I., "Bare Tethers for Electrodynamic Spacecraft Propulsion," Journal of Spacecraft and Rockets, Vol. 37, No. 2, March-April 2000, pp. $205-211$.

${ }^{3}$ Vasil'yev, Y. V. and V. V. Danilov, "Proposal for an Attempt to Influence the Precipitation of Charged Particles from the Earth's Radiation Belts by Means of a Satellite-Borne High-Voltage Cable System," Transaction (Doklady) of the Russian Academy of Sciences: Earth Science Sections, Vol. 345A, No. 9, October 1996, pp. 172-176.

${ }^{4}$ V. V. Danilov, V. V. Mirnov, and Ucer, D., "High-Voltage Space Tether for Enhanced Particle Scattering in Van Allen Belts," Fusion Technology, Vol. 35, No. 1T, January 1999, pp. 312-314.

${ }^{5}$ V. V. Danilov, Elgin, B., Grafodatsky, O., and Mirnov, V., "High-Voltage Satellite Tethers For Active Experiments In Space," Proceedings of the 6th Spacecraft Charging Technology Conference, AFRL-VS-TR-20001578, September 2000, pp. $165-168$.

${ }^{6}$ Choinière, É., Theory and Experimental Evaluation of a Consistent Steady-State Kinetic Model for 2-D Conductive Structures in Ionospheric Plasmas with Application to Bare Electrodynamic Tethers in Space, Ph.D. thesis, University of Michigan, 2004.

${ }^{7}$ Laframboise, J., Theory of Spherical and Cylindrical Langmuir Probes in a Collisionless, Maxwellian Plasma at Rest, Ph.D. thesis, University of Toronto, June 1966.

${ }^{8} \mathrm{Xu}, \mathrm{G} . \mathrm{Z} .$, The Interation of a Moving Spacecraft with the Ionosphere: Current Collection and Wake Structure, Ph.D. thesis, York University, 1992.

${ }^{9}$ McMahon, J. C., The Interaction of Infinite and Finite Cylindrical Probes with a Drifting Collisionless Maxwellian Plasma, Ph.D. thesis, York University, September 2000.

${ }^{10}$ É. Choinière and Gilchrist, B. E., "Modeling Long Probes in Flowing Plasmas using KiPS-2D, a Novel Steady-State Vlasov Solver," AIAA Paper AIAA-2003-5098, 2003.

${ }^{11}$ Cheng, D. K., Field and Wave Electromagnetics, Addison-Wesley Pub. Co., 1989.

${ }^{12}$ Lieberman, M. A. and Lichtenberg, A. J., Principles of Plasma Discharges and Materials Processing, Wiley Interscience, 1994.

${ }^{13}$ Parker, L. W., "Computation of Collisionless Steady-State Plasma Flow Past a Charged Disk," Tech. rep., Lee W. Parker, Inc., 1976, Prepared for NASA - George C. Marshall Space Flight Center.

${ }^{14}$ Tong, P. and Rossettos, J. N., Finite-Element Method - Basic Technique and Implementation, chap. 2: The FiniteElement Method for Poisson's Equation, The MIT Press, 1977, pp. 40-71.

${ }^{15}$ Evans, L. C., Partial Differential Equations, American Mathematical Society, 1998.

${ }^{16}$ Sikorski, K. A., Optimal Solution of Nonlinear Equations, Oxford University Press, 2001.

${ }^{17}$ Burden, R. L., Numerical Analysis, PWS-KENT Publishing Company, 4th ed., 1989.

${ }^{18}$ Geist, A., Beguelin, A., Dongarra, J., Jiang, W., Manchek, R., and Sunderam, V., PVM 3 User's Guide and Reference Manual, Oak Ridge National Laboratory, September 1994.

${ }^{19}$ Estes, R. and Sanmartín, J., "Cylindrical Langmuir probes beyond the orbital-motion-limited regime," Physics of Plasmas, Vol. 7, No. 10, October 2000, pp. 4320-4325.

${ }^{20}$ Sanmartín, J. and Estes, R., "Interference of Parallel Cylindrical Langmuir Probes," Physics of Plasmas, Vol. 8, No. 9, September 2001, pp. 4234-4239.

${ }^{21}$ Choinière, É., Gilchrist, B. E., Bilén, S. G., Furhrhop, K. R., and Gallimore, A. D., "Experimental Investigation of Electron Collection to Solid and Slotted Tape Probes in a High-Speed Flowing Plasma," IEEE Transactions on Plasma Science, 2004, submitted for publication in April 2004.
} 


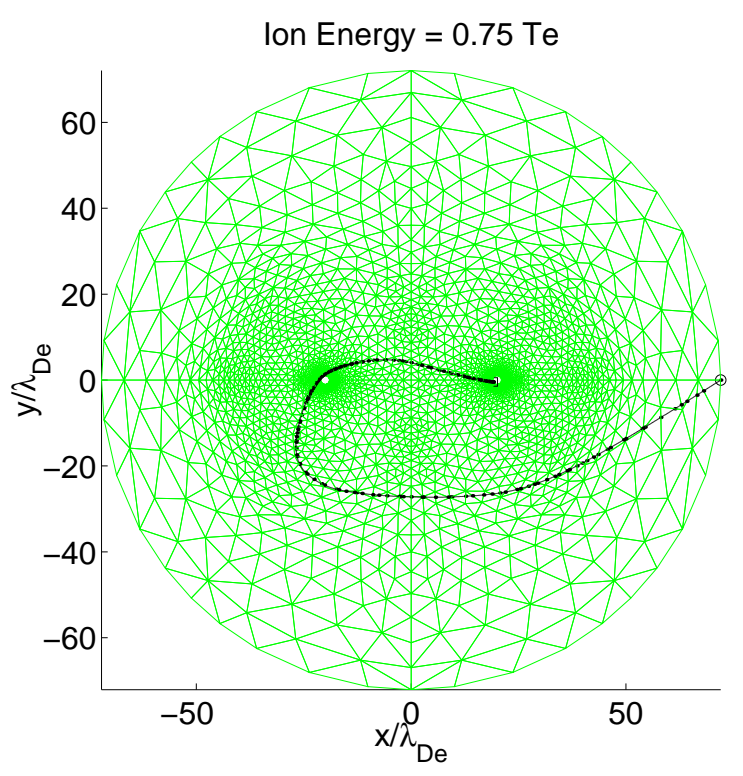

(a) Unpopulated Orbit Originating From Cylinder Surface

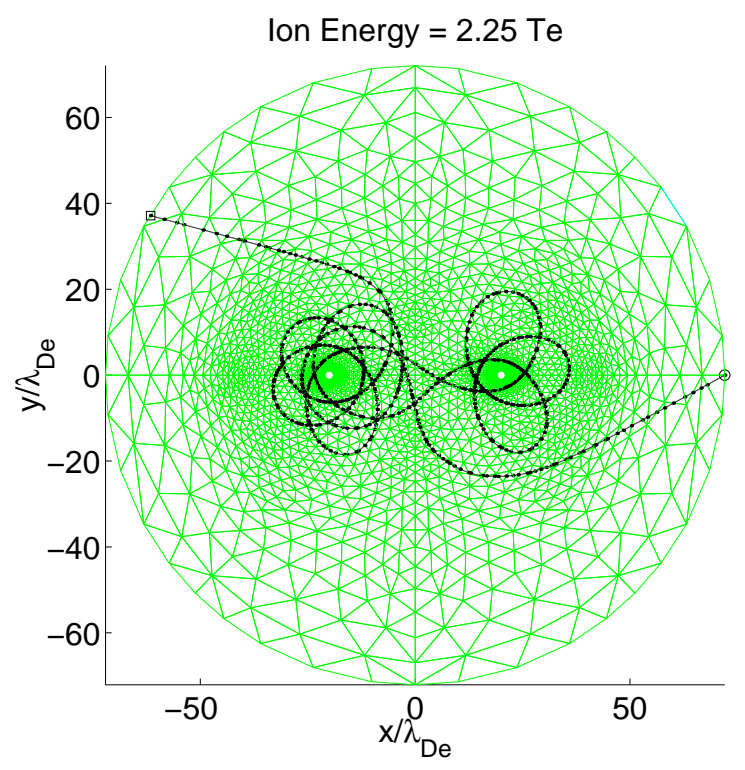

(c) Complex Populated Orbit Around Both Cylinders

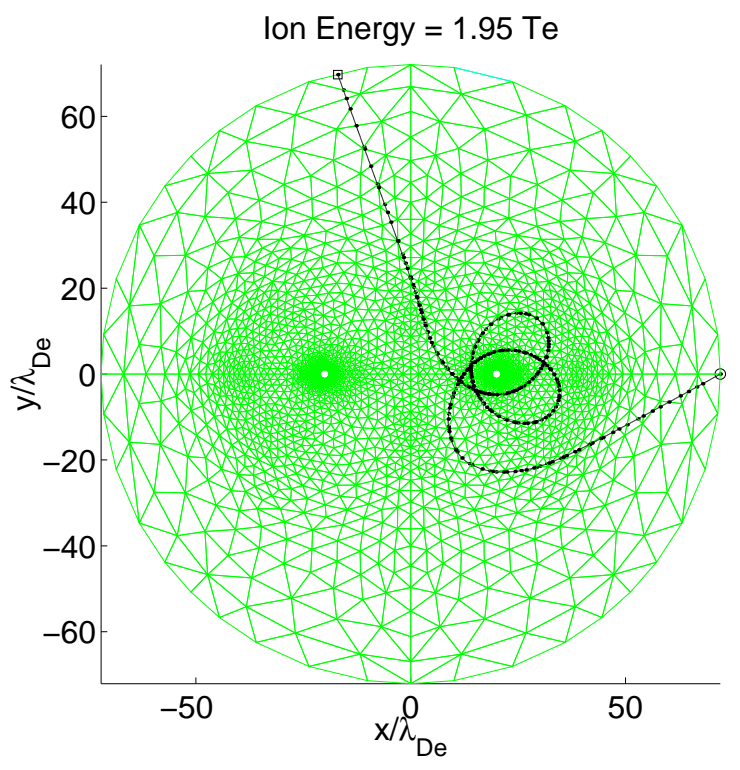

(b) Complex Populated Orbit Around One Cylinder

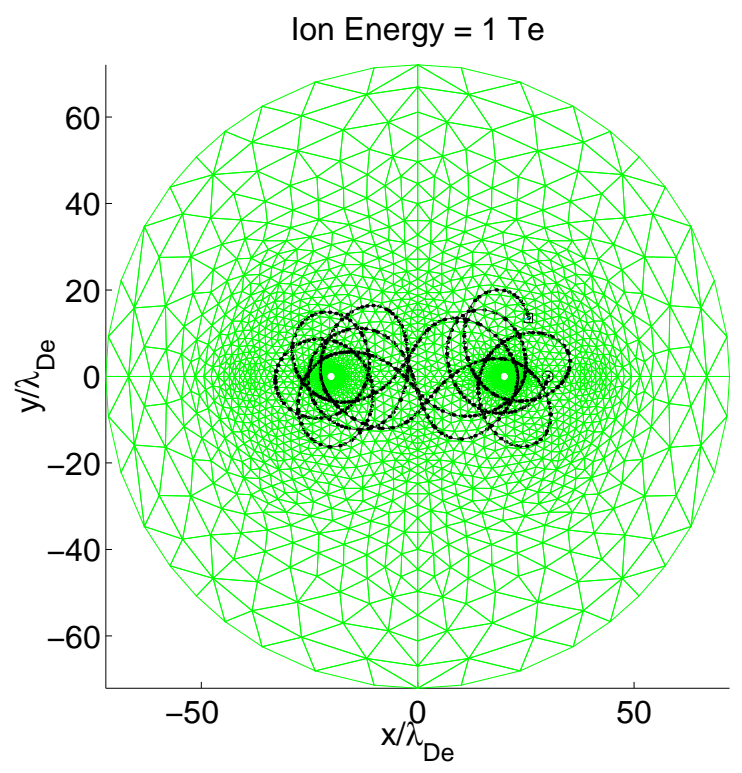

(d) Complex Unpopulated Orbit Around Both Cylinders

Figure 3. Examples of some typical ion orbits within the self-consistent potential structure of a two-cylinder system. The square and circle indicate the source and interrogation points of the orbits, respectively. The total ion energy (potential plus kinetic) is indicated above each plot in terms of the electron temperature (in units of $\mathrm{eV}$ ). Intersections with the background mesh are marked with dots. 

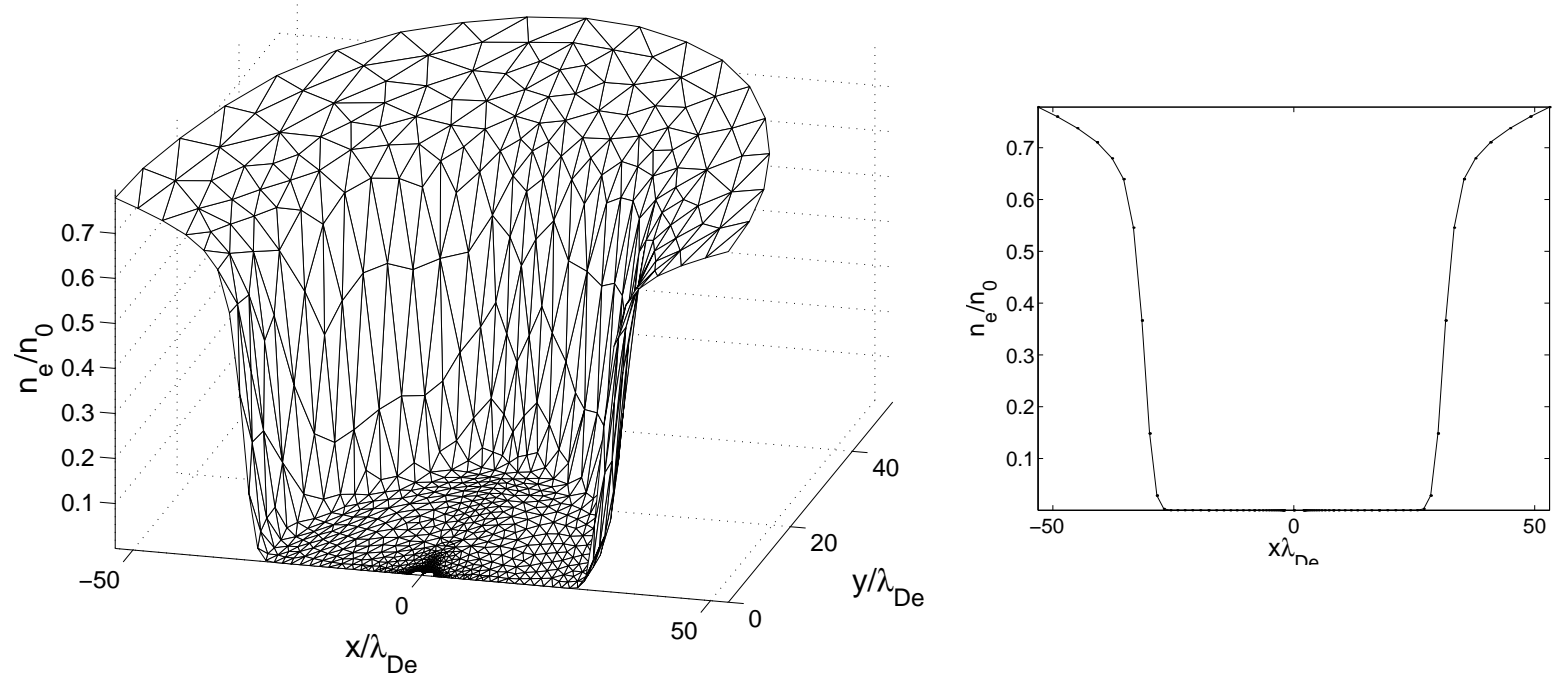

(a) Normalized Electron Number Density $n_{e} / n_{0}$.
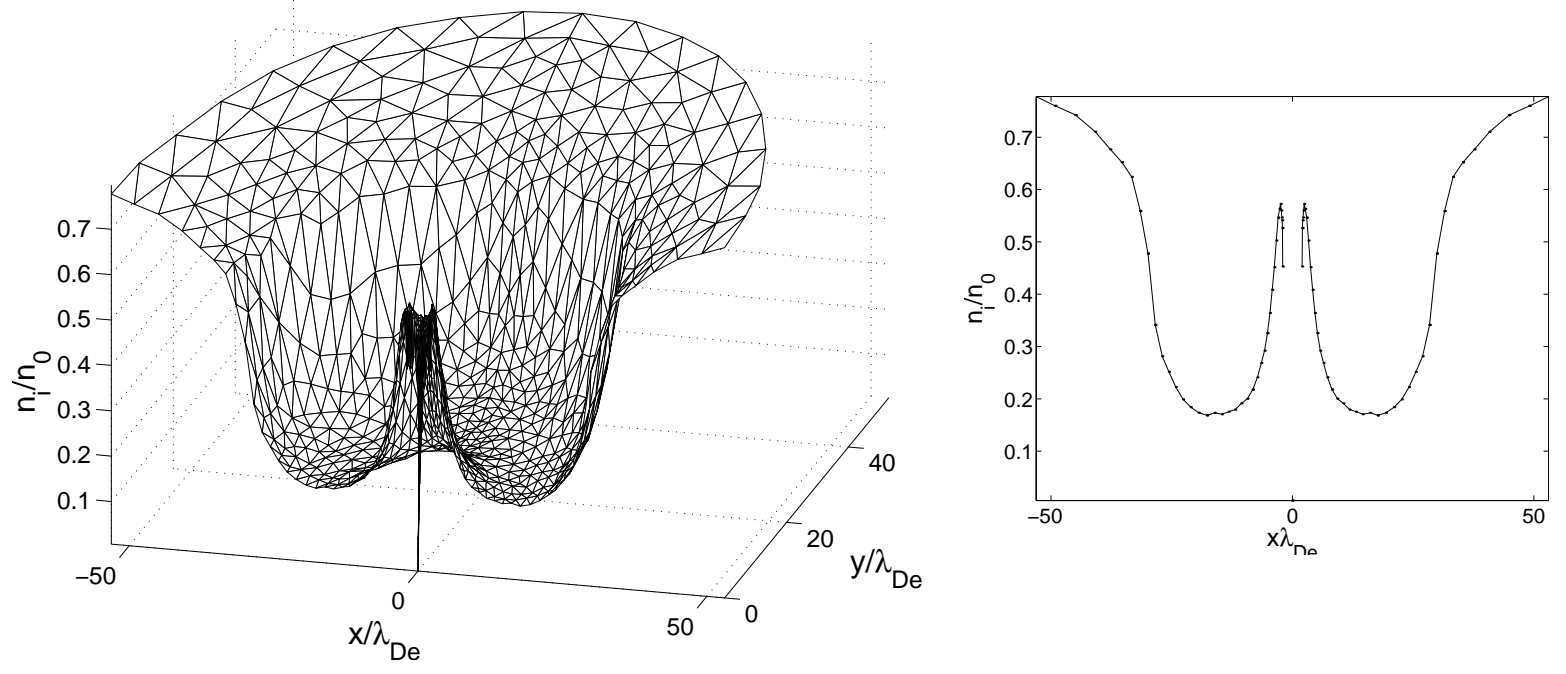

(b) Normalized Ion Number Density $n_{i} / n_{0}$.

Figure 4. Self-consistent electron and ion density distributions for the two-cylinder configuration with a cylinder radius $r_{0}=\lambda_{\text {De }}$, center-to-center spacing $\Delta x=2 \lambda_{\mathrm{De}}$, and normalized bias $\phi_{0}=-320$. These density profiles constitute the output of the Vlasov solver and are subtracted to form the output net charge density profile $\vec{\rho}_{\text {out }}=e\left(n_{i}-n_{e}\right)$ depicted on Figure 1 . 

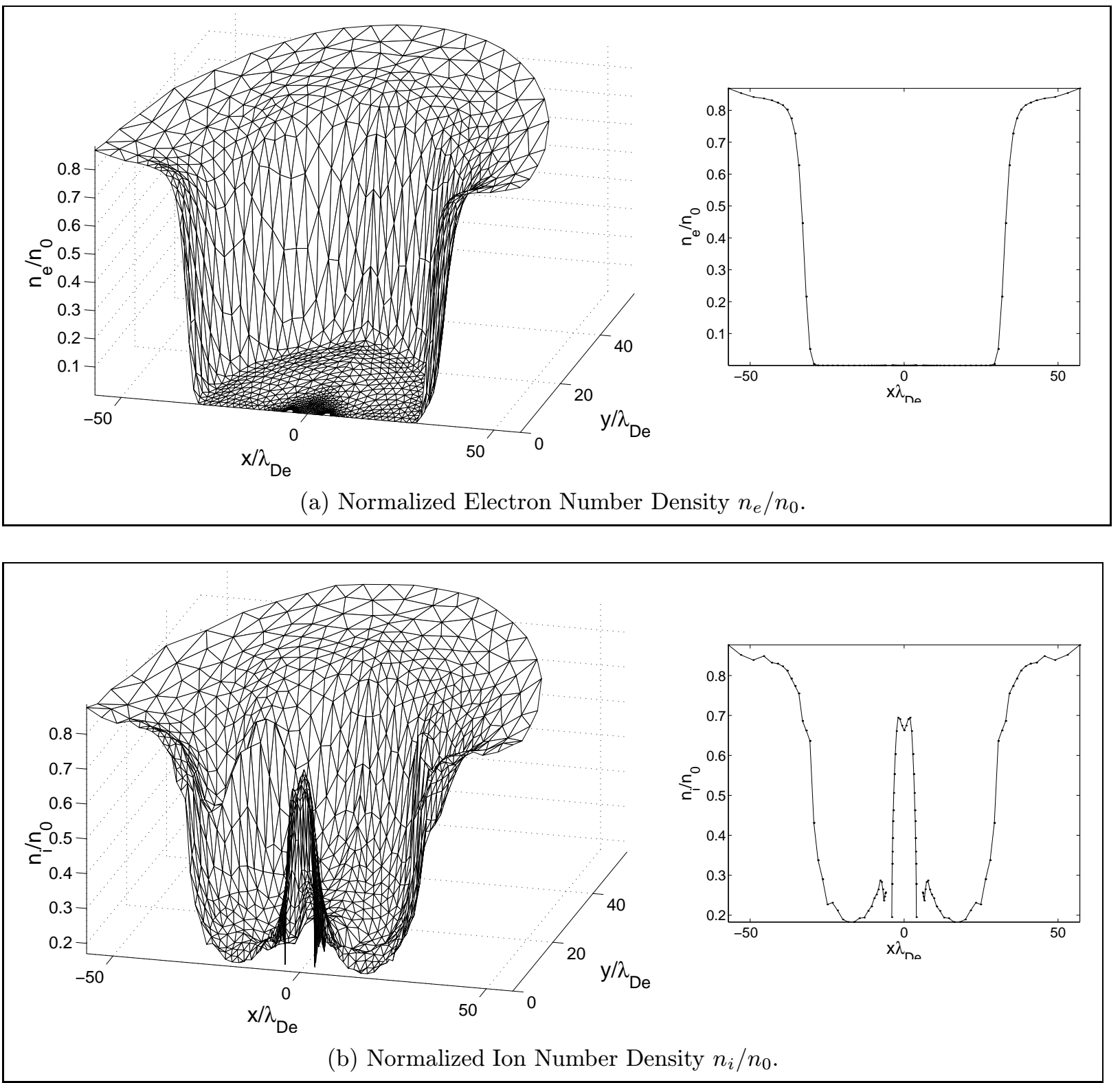

Figure 5. Self-consistent electron and ion density distributions for the two-cylinder configuration with a cylinder radius $r_{0}=\lambda_{\text {De }}$, center-to-center spacing $\Delta x=10 \lambda_{\text {De }}$, and normalized bias $\phi_{0}=-320$. These density profiles constitute the output of the Vlasov solver and are subtracted to form the output net charge density profile $\vec{\rho}_{\text {out }}=e\left(n_{i}-n_{e}\right)$ depicted on Figure 1 . 

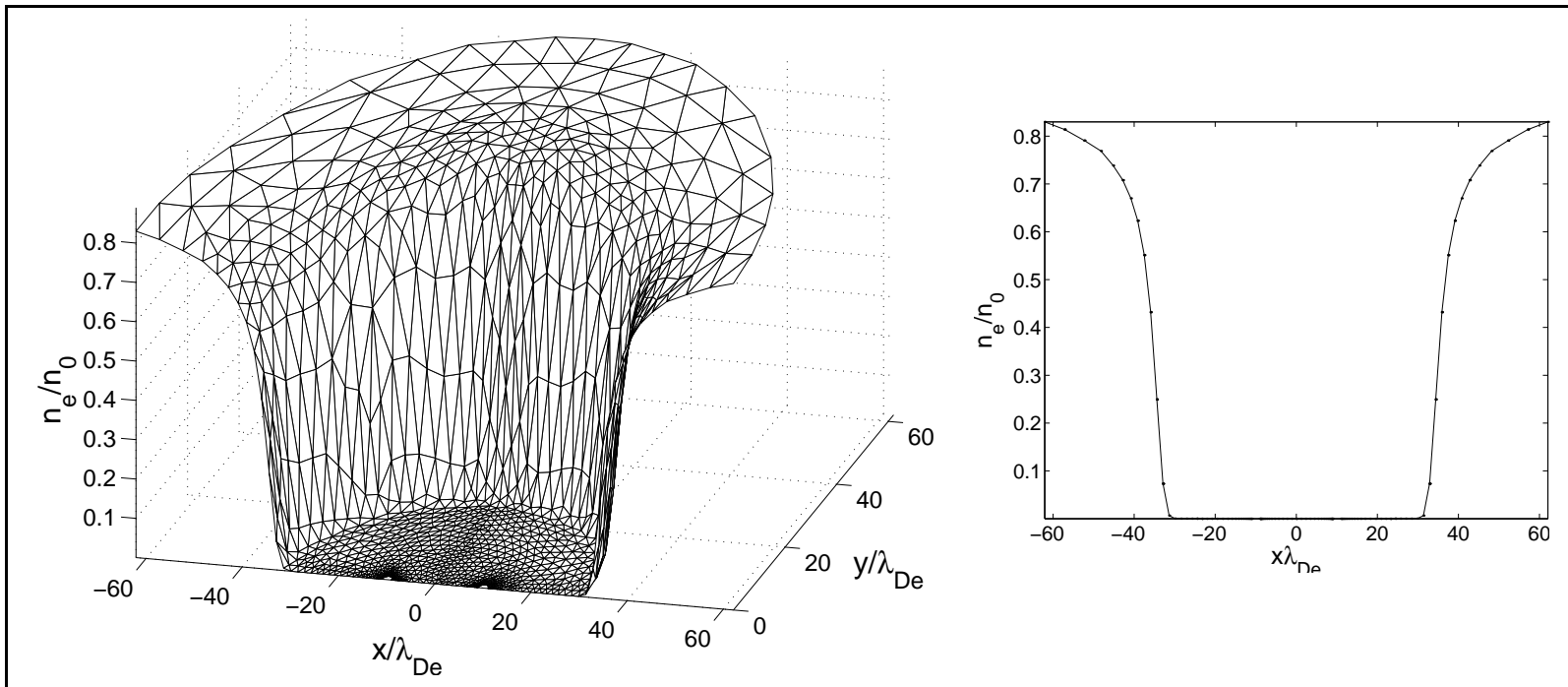

(a) Normalized Electron Number Density $n_{e} / n_{0}$.
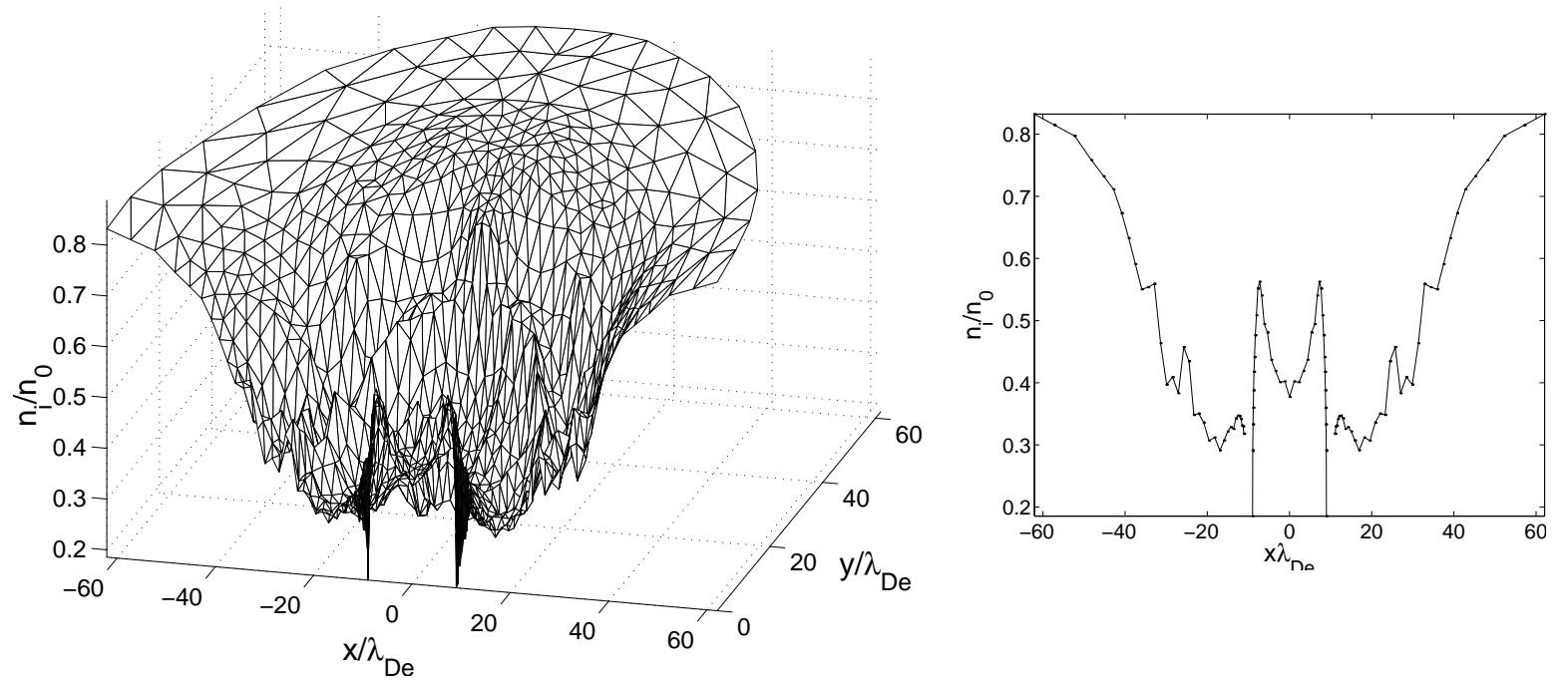

(b) Normalized Ion Number Density $n_{i} / n_{0}$.

Figure 6. Self-consistent electron and ion density distributions for the two-cylinder configuration with a cylinder radius $r_{0}=\lambda_{\text {De }}$, center-to-center spacing $\Delta x=20 \lambda_{\text {De }}$, and normalized bias $\phi_{0}=-320$. These density profiles constitute the output of the Vlasov solver and are subtracted to form the output net charge density profile $\vec{\rho}_{\text {out }}=e\left(n_{i}-n_{e}\right)$ depicted on Figure 1 . 

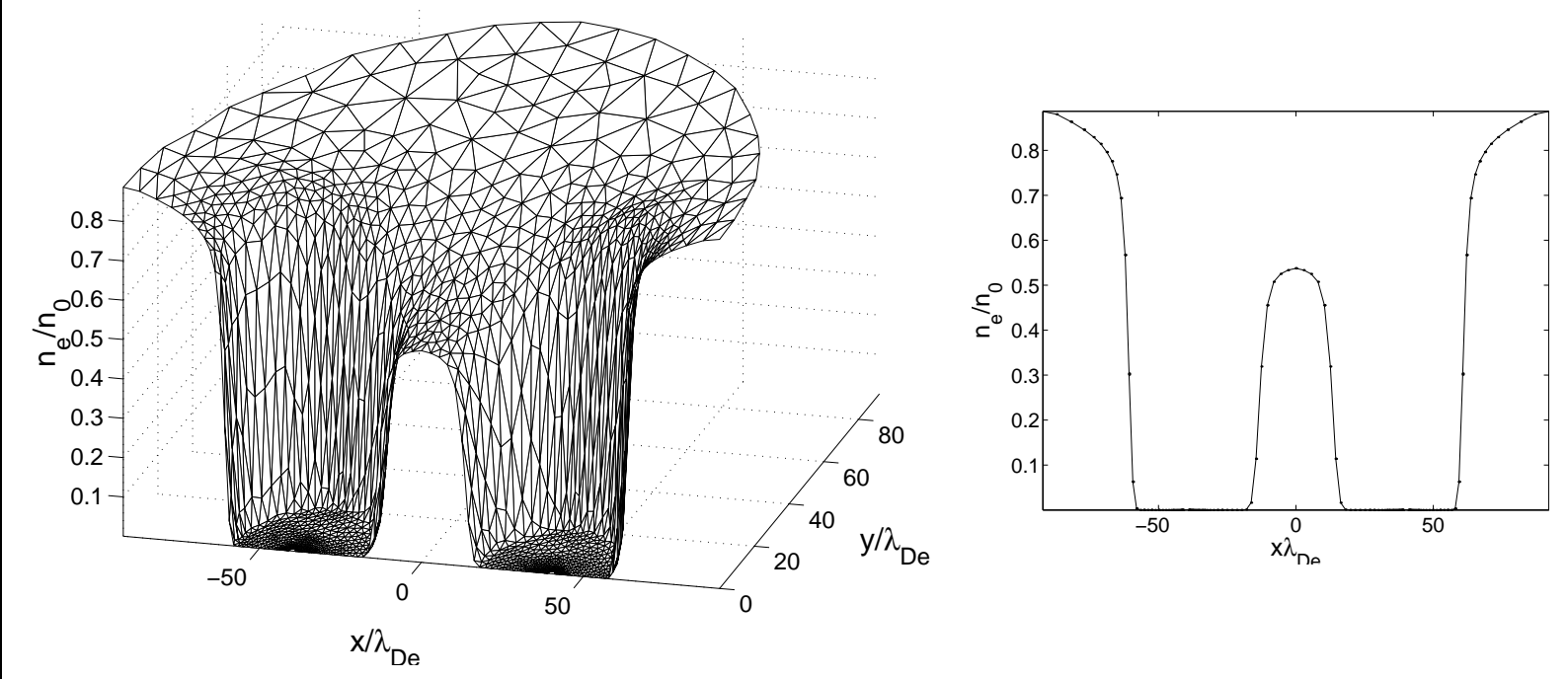

(a) Normalized Electron Number Density $n_{e} / n_{0}$.
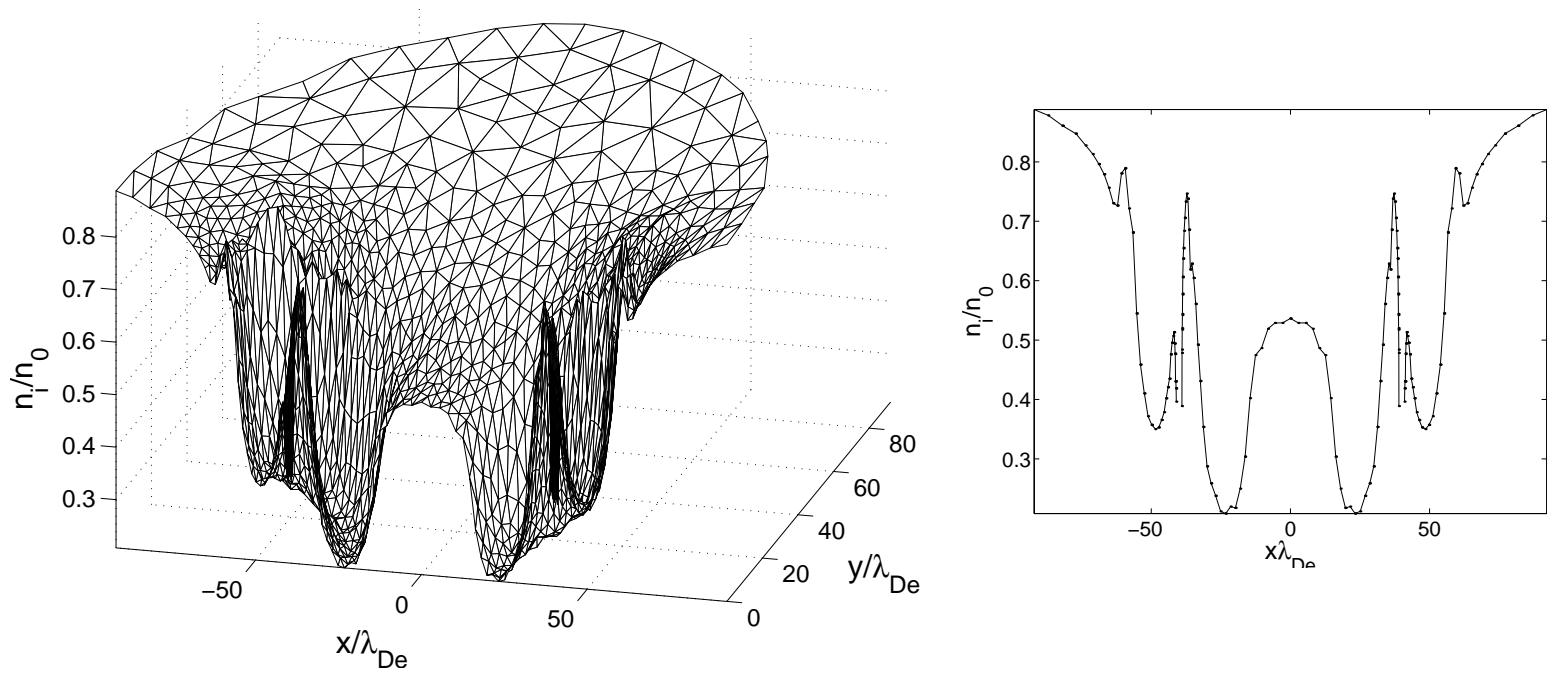

(b) Normalized Ion Number Density $n_{i} / n_{0}$.

Figure 7. Self-consistent electron and ion density distributions for the two-cylinder configuration with a cylinder radius $r_{0}=\lambda_{\text {De }}$, center-to-center spacing $\Delta x=80 \lambda_{\text {De }}$, and normalized bias $\phi_{0}=-320$. These density profiles constitute the output of the Vlasov solver and are subtracted to form the output net charge density profile $\vec{\rho}_{\text {out }}=e\left(n_{i}-n_{e}\right)$ depicted on Figure 1 . 


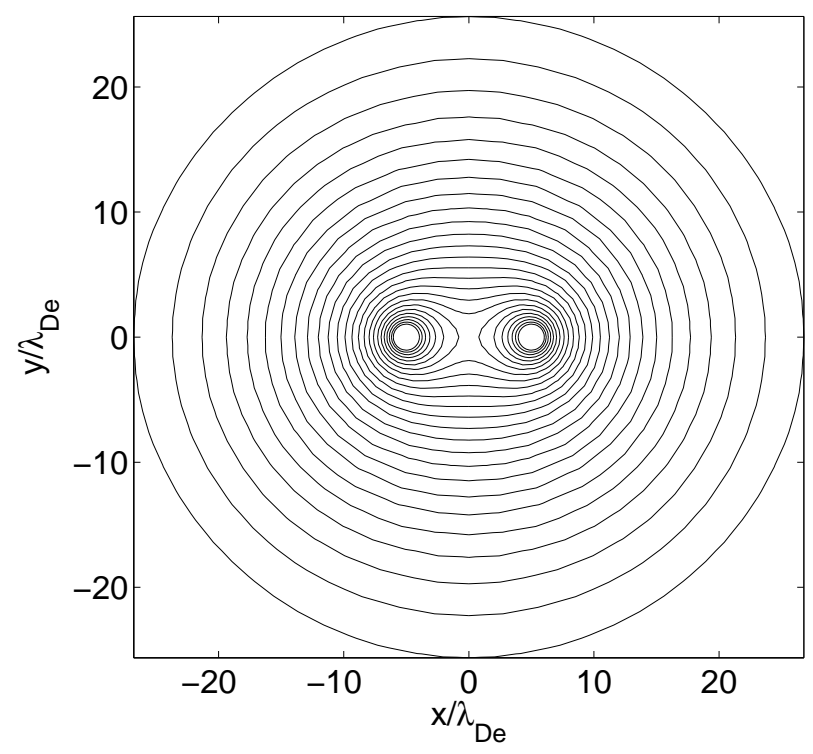

(a) Contour Levels

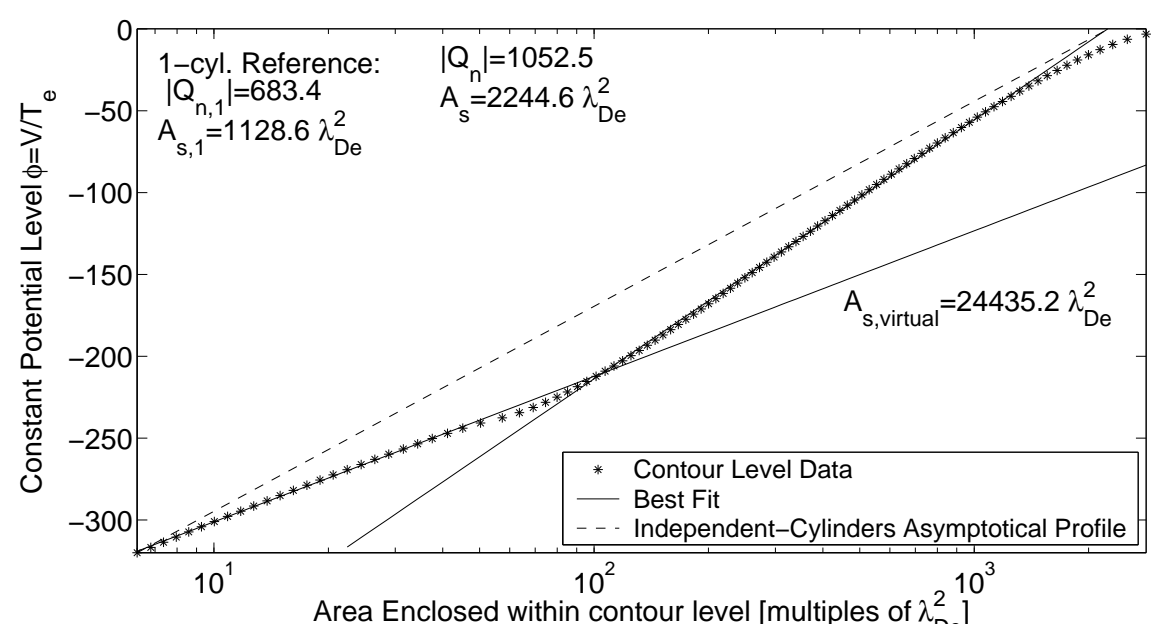

(b) Potential vs Enclosed Area

Figure 8. Potential contour levels (a) along with a plot of the enclosed area as a function of the level of a set contour levels of the potential structure (b) for two cylinders with a center-to-center spacing $\Delta x=10 \lambda_{\mathrm{De}}$. The cylinder radius is $r_{0}=\lambda_{\mathrm{De}}$ and both cylinders are biased at a normalized potential $\phi_{0}=-320$. 


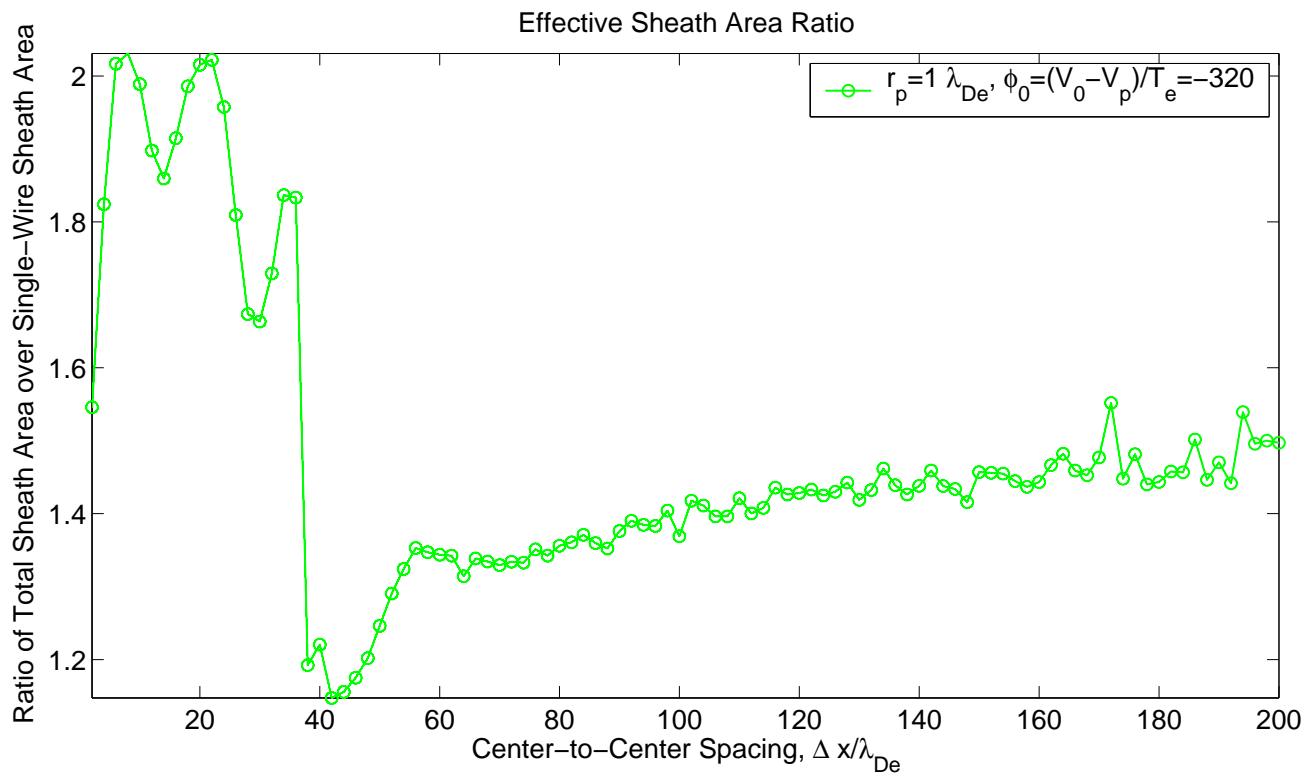

Figure 9. Effective sheath area ratio as a function of center-to-center spacing of two parallel cylinders. The results shown here apply to the case of two cylinders of radius $r_{0}=\lambda_{\text {De }}$ with normalized bias $\phi_{0}=-320$. The effective sheath area ratio is the ratio of the two-cylinder sheath area to the single-cylinder sheath area.

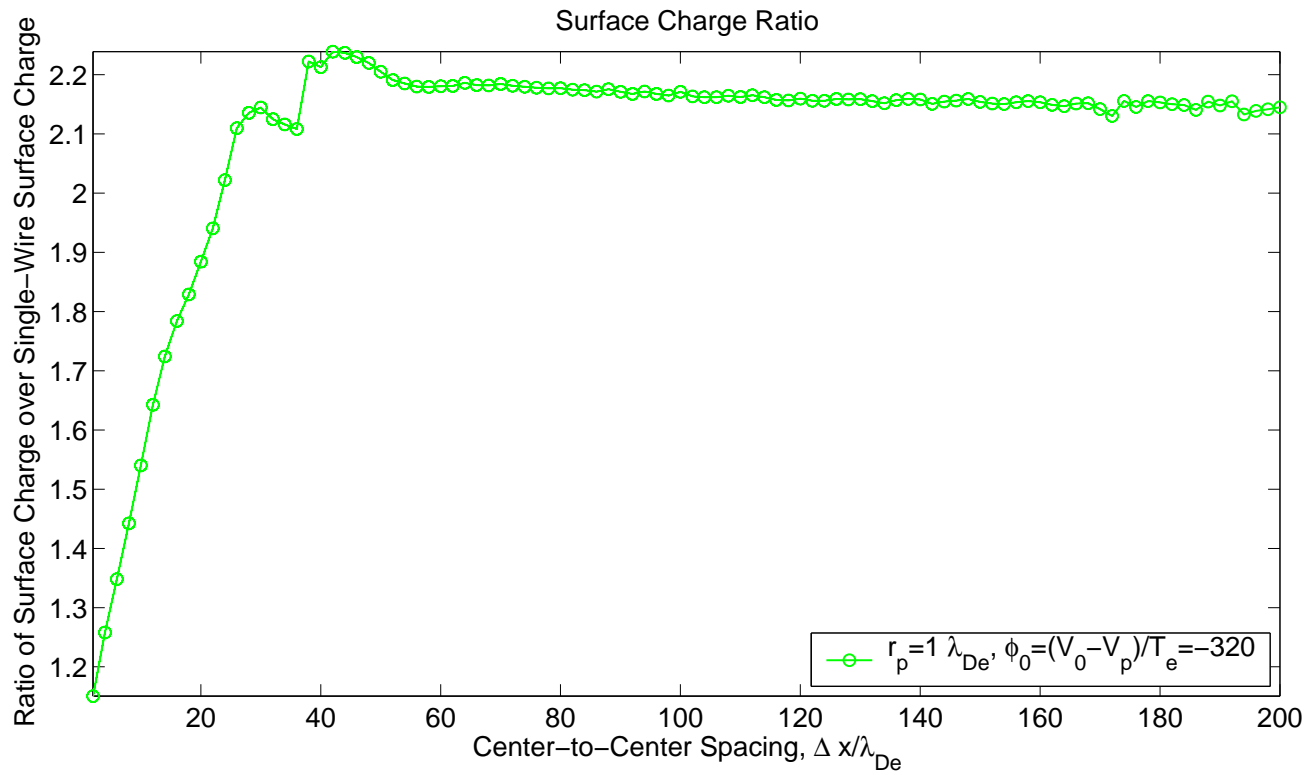

Figure 10. Ratio of the total surface charge on both cylinders to the surface charge held by a single independent cylinder. The results shown here apply to the case of two cylinders of radius $r_{0}=\lambda_{\text {De }}$ with normalized bias $\phi_{0}=-320$. 


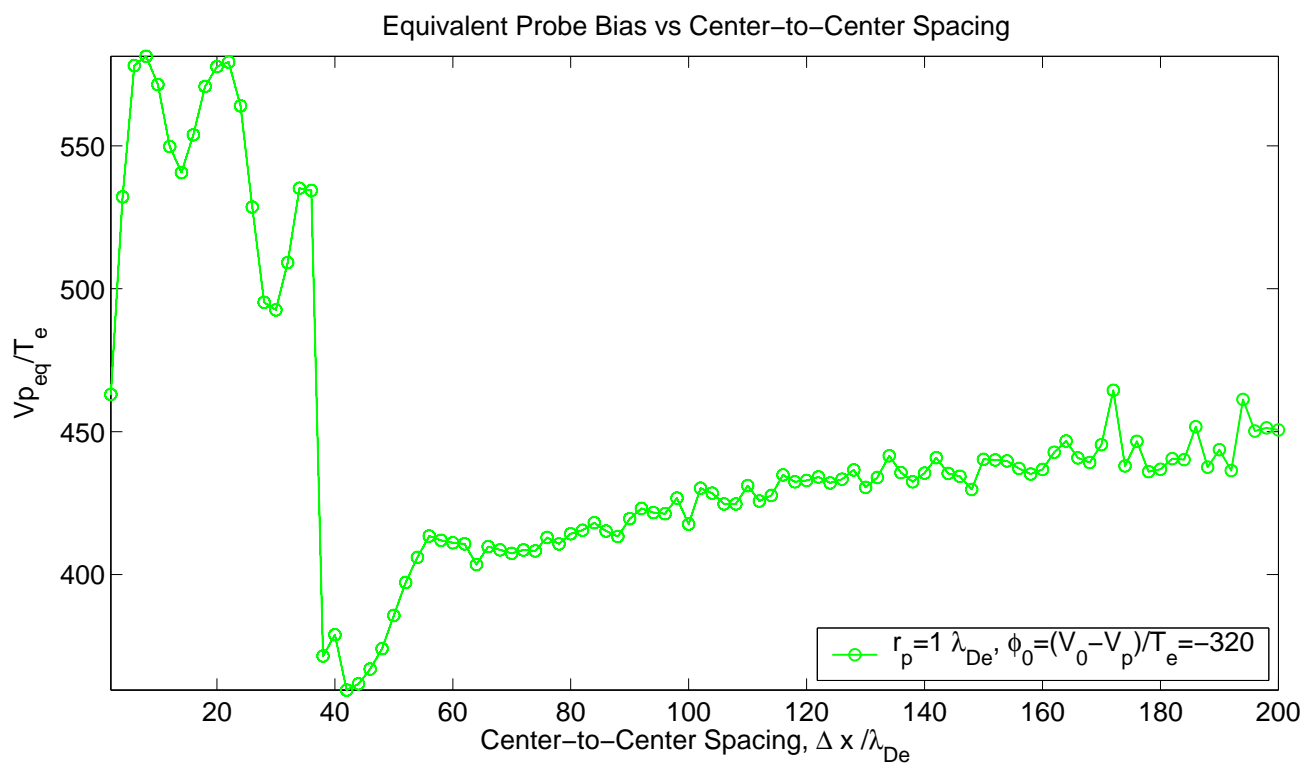

Figure 11. Equivalent bias of a single cylinder as a function of the center-to-center spacing of two parallel cylinders. The results shown here apply to the case of two cylinders of radius $r_{0}=\lambda_{\text {De }}$ with normalized bias $\phi_{0}=-320$. The equivalent bias is that which would be necessary for a single cylinder to yield the same sheath area as the combination of the two cylinders.

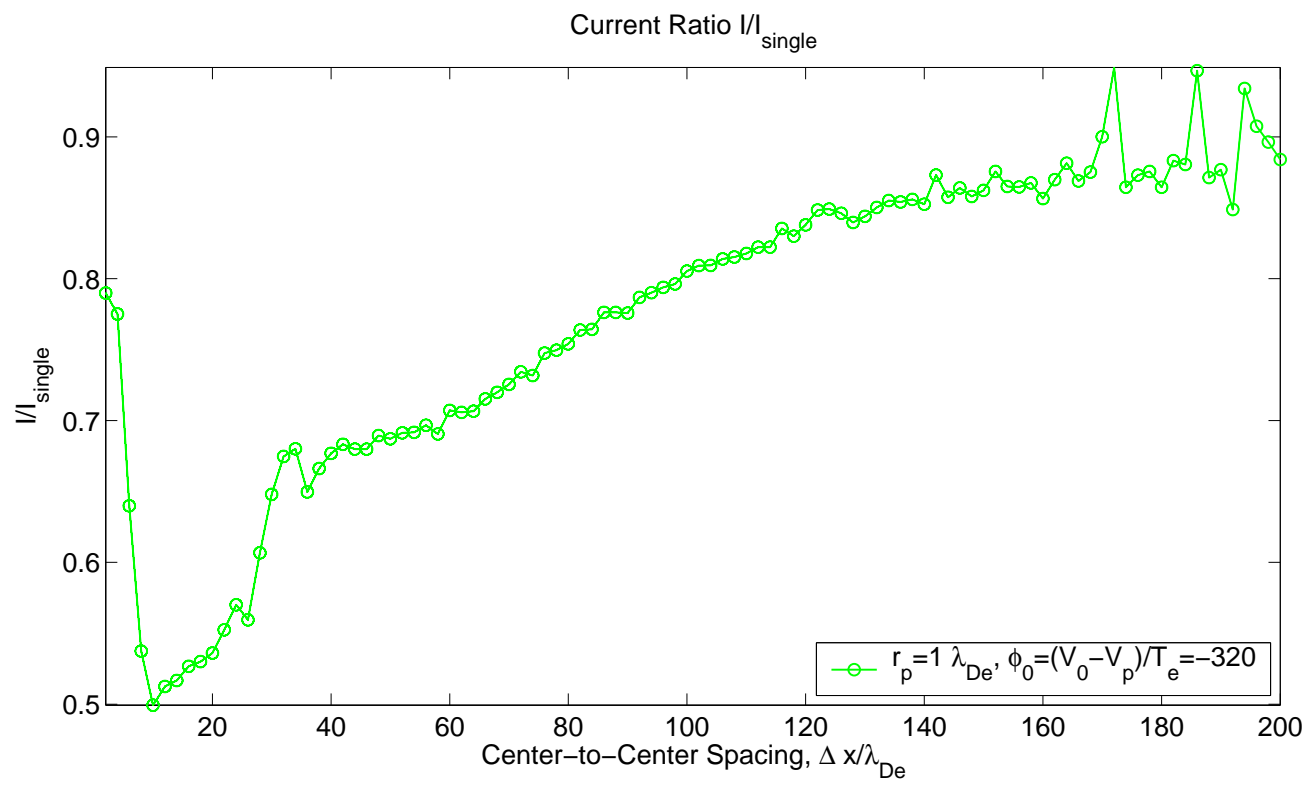

Figure 12. Current ratio as a function of center-to-center spacing for a two-cylinder configuration. The current ratio is defined as the ratio of the total collected current to the current that would be collected by two independent cylinders. 